\title{
An algorithm for finding parameters of tunnels
}

\author{
KAI ISHIHARA
}

Cho and McCullough gave a numerical parameterization of the collection of all tunnels of all tunnel number 1 knots and links in the 3-sphere. Here we give an algorithm for finding the parameter of a given tunnel by using its Heegaard diagram.

$57 \mathrm{M} 25 ; 57 \mathrm{~N} 10$

\section{Introduction}

Pairs consisting of a tunnel number one knot (or link) and one of its unknotting tunnels can be identified with isotopy classes of nonseparating (or separating) essential disks in a standard genus-2 handlebody $H$ in $S^{3}$. That is, the equivalence classes of such tunnels up to orientation-preserving homeomorphism in $S^{3}$ can be identified with the vertices of the quotient of the disk complex of $H$ by the action of the Goeritz group of the pair $\left(S^{3}, H\right)$. In this quotient, Cho and McCullough [3] defined a principal path for each tunnel. The existence of the principal path implies that every tunnel can be obtained by starting from the unique tunnel of the trivial knot and performing a uniquely determined sequence of simple constructions. Each construction is determined by a rational number, which is essentially a Scharlemann and Thompson invariant [12]. In other words, a tunnel of a tunnel number 1 knot or link is determined by a parameter consisting of a sequence of rational invariants and some additional data that encodes the path. Thus by comparing the parameters of two tunnels, we can decide whether or not they are equivalent. The parameter contains other information about the tunnel as well. In particular, it tells whether or not the tunnel is a $(1,1)$-tunnel, that is, an upper tunnel associated to some genus-1 1-bridge position of the knot.

Cho and McCullough computed the parameters for all tunnels of 2-bridge knots [3], and for all tunnels of torus knots [2]. In this paper, we will prove the following theorem.

Theorem 1.1 There is an algorithm for finding the parameter of a tunnel given as a disk in a standard genus-2 handlebody in $S^{3}$. 
The algorithm uses Heegaard diagrams. Section 4 describes the relation between the Heegaard diagram and the link diagram of an unknotting tunnel. In Section 5, we will show how to calculate the rational invariant for each step in the principal path, and in Sections 6 and 7, we give the algorithm and its proof. In Section 8, we apply the algorithm to find the principal paths of two tunnels of the twisted torus knot $T(5,7,2)$ found by Goda and Hayashi. One of the tunnels is known to be a $(1,1)-$ tunnel, and from the principal path, we deduce that the other is not a $(1,1)$-tunnel. This provides the first known example, other than torus knots, of a knot having both $(1,1)$ and non-(1,1)-tunnels.

Acknowledgments I gratefully acknowledge much helpful advice and constant encouragement of Professor Koya Shimokawa. I am greatly indebted to thank Professor Hiroshi Goda, who suggested the application of the algorithm in Section 8. I also would like to thank to the referee for a careful reading of the paper and helpful comments. This research is partially supported by EPSRC Grant EP/H031367/1.

\section{Preliminaries}

In this section we present some definitions. See [3] for details.

Closure, interior and regular neighborhood are denoted by $\mathrm{Cl}(\cdot), \operatorname{Int}(\cdot)$ and $\mathrm{N}(\cdot)$ respectively throughout this paper. We use the same notation as in [3]: $H$ is the standard genus-2 handlebody in $S^{3}, \mathcal{K}(H)$ is the disk complex of $H, \mathcal{D}(H)$ is the subcomplex of $\mathcal{K}(H)$ spanned by vertices corresponding to nonseparating essential properly embedded disks in $H, \mathcal{D}^{\prime}(H)$ is the first barycentric subdivision of $\mathcal{D}(H)$, $\widetilde{\mathcal{T}}$ is the subcomplex of $\mathcal{D}^{\prime}(H)$ obtained by removing the open stars of the vertices of $\mathcal{D}(H), \mathcal{G}$ is the Goeritz group that is the group of isotopy classes of orientationpreserving diffeomorphisms of $S^{3}$ that take $H$ onto itself, and $\mathcal{T}$ is the quotient of $\tilde{\mathcal{T}}$ by the action of $\mathcal{G}$.

Definition 2.1 A tunnel is a $\mathcal{G}$-orbit of disks in $H$. In particular, a $\mathcal{G}$-orbit of nonseparating disks in $H$ is called a knot tunnel, and a $\mathcal{G}$-orbit of separating disks in $H$ is called a link tunnel.

Thus the knot tunnels correspond exactly to the vertices of the quotient $\mathcal{D}(H) / \mathcal{G}$ of $\mathcal{D}(H)$ by the action of $\mathcal{G}$. If $\tau$ is a knot tunnel, it is a tunnel of the knot $K_{\tau}$ that is a core of the solid torus obtained by cutting $H$ along $\tau$. If $\tau$ is a link tunnel, it is a tunnel of the link $K_{\tau}$ that consists of cores of the two solid tori obtained by cutting $H$ along $\tau$. We regard $K_{\tau}$ as defined only up to isotopy in $S^{3}$. 
A disk $D$ in $H$ is primitive if there exists a properly embedded disk $D^{\prime}$ in the complementary handlebody $\mathrm{Cl}\left(S^{3}-H\right)$ such that the circles $\partial D$ and $\partial D^{\prime}$ in $\partial H$ intersect transversely in a single point. Viewed as tunnels, primitive disks are exactly the tunnels of the trivial knot, and are all equivalent.

Definition 2.2 A primitive pair is a pair of disjoint nonisotopic primitive disks and a primitive triple is a triple of pairwise disjoint nonisotopic primitive disks.

Cho and McCullough showed that $\tilde{\mathcal{T}}$ and $\mathcal{T}$ are trees. There is a vertex $\theta_{0}$ that is the unique $\mathcal{G}$-orbit of a primitive triples. The principal path of a tunnel, that will become the foundation for the parameter, can be defined as follows. Let $D$ be a disk in $H$. If $D$ is nonseparating, then there are two isotopy classes of nonseparating disks $D_{1}$ and $D_{2}$ such that among all triples having $D$ as a member, the triple $\left\{D, D_{1}, D_{2}\right\}$ is closest in $\tilde{\mathcal{T}}$ to the tree spanned by the primitive triples. If $D$ is separating, $D$ is disjoint from only two isotopy classes of nonseparating disks, so is a vertex of only one 2-simplex $\left\{D, D_{1}, D_{2}\right\}$, which is attached to $\mathcal{D}(H)$ along $\left\{D_{1}, D_{2}\right\}$. Let $\tau$ be the tunnel that is the $\mathcal{G}$-orbit of $D$.

Definition 2.3 The principal vertex of $\tau$ is the $\mathcal{G}$-orbit of $\left\{D, D_{1}, D_{2}\right\}$. The principal pair of $\tau$ is the $\mathcal{G}$-orbit of $\left\{D_{1}, D_{2}\right\}$. The principal path of $\tau$ is the unique path in $\mathcal{T}$ from $\theta_{0}$ to the principal vertex of $\tau$.

Remark 2.4 The principal pairs of tunnels and the principal triples of knot tunnels are in $\mathcal{T}$, but tunnels and principal vertices of link tunnels are not.

Let $\mu$ be a pair of disjoint nonisotopic nonseparating disks in $H$. A disk in $H$ disjoint from $\mu$ and not parallel to either of them is called slope disk for $\mu$. Let $\tau$ be a nonseparating slope disk for $\mu$.

Definition 2.5 The slope 0 perpendicular disk $\tau^{0}$ for $(\mu ; \tau)$ is defined by the following properties:

(1) $\tau^{0}$ is slope disk for $\mu$.

(2) $\tau$ and $\tau^{0}$ intersect transversely in one arc.

(3) $\tau^{0}$ separates $H$ (and so $K_{\tau^{0}}$ is a two-component link).

(4) The linking number of $K_{\tau^{0}}$ is 0 .

Then $\tau$ and $\tau^{0}$ can be a coordinate system, and a rational number is naturally defined for each slope disk for $\mu$, that is called its $(\mu ; \tau)-$ slope (see [3, Section 8]). 
Theorem 2.6 [3, Theorem 12.3] Let $\tau$ be a tunnel with principal path $\theta_{0}, \mu_{0}$, $\mu_{0} \cup\left\{\tau_{0}\right\}, \mu_{1}, \ldots, \mu_{n}, \mu_{n} \cup\left\{\tau_{n}\right\}$. Fix a lift of the principal path to $\mathcal{D}(H)$, so that each $\mu_{i}$ corresponds to an actual pair of disks in $H$.

(1) If $\tau$ is primitive, put $m_{0}=[0] \in \mathbb{Q} / \mathbb{Z}$. Otherwise, let $m_{0}=\left[p_{0} / q_{0}\right] \in Q / Z$ be the simple slope of $\tau_{0}$.

(2) If $n \geq 1$, then for $1 \leq i \leq n$ let $\sigma_{i}$ be the unique disk in $\mu_{i-1}-\mu_{i}$ and let $m_{i}=q_{i} / p_{i} \in \mathbb{Q}$ be the $\left(\mu_{i} ; \sigma_{i}\right)$-slope of $\tau_{i}$.

(3) If $n \geq 2$, then for $2 \leq i \leq n$ define $s_{i}=0$ or $s_{i}=1$ according to whether or not the unique disk of $\mu_{i} \cap \mu_{i-1}$ equals the unique disk of $\mu_{i-1} \cap \mu_{i-2}$.

Then, sending $\tau$ to the pair $\left(\left(m_{0}, \ldots, m_{n}\right),\left(s_{2}, \ldots, s_{n}\right)\right)$ is a bijection from the set of all tunnels to the set of all elements $\left(\left(\left[p_{0} / q_{0}\right], q_{1} / p_{1}, \ldots, q_{n} / p_{n}\right),\left(s_{2}, \ldots, s_{n}\right)\right)$ in

$$
(\mathbb{Q} / \mathbb{Z}) \cup(\mathbb{Q} / \mathbb{Z} \times \mathbb{Q}) \cup\left(\bigcup_{n \geq 2} \mathbb{Q} / \mathbb{Z} \times \mathbb{Q}^{n} \times\{0,1\}^{n-2}\right),
$$

with $q_{i}$ odd for $1 \leq i \leq n-1$.

Note that $q_{n}$ is odd if and only if $\tau$ is a knot tunnel.

Definition 2.7 We call $\left(\left(\left[p_{0} / q_{0}\right], q_{1} / p_{1}, q_{2} / p_{2}, \ldots, q_{n} / p_{n}\right),\left(s_{2}, \ldots, s_{n}\right)\right)$ the $p a$ rameter of $\tau$. The numbers $s_{2}, \ldots, s_{n}$ are called the binary invariants of $\tau$.

Next we present a definition of the depth, for use in Section 8.

Definition 2.8 The depth of a tunnel $\tau$, which is denoted by depth $(\tau)$, is the simplicial distance in the 1 -skeleton of $\mathcal{D}(H) / \mathcal{G}$ from $\tau$ to the primitive vertex $\pi_{0}$.

Remark 2.9 A tunnel $\tau$ of nontrivial knot is a $(1,1)$-tunnel if and only if depth $(\tau)=1$.

Hence we can determine whether or not a tunnel is $(1,1)$ from its depth. In [4], Cho and McCullough showed how to compute the depth from the binary invariants.

Proposition 2.10 [4] Let $\tau$ be a knot tunnel and $s_{2}, \ldots, s_{n}$ the binary invariants of $\tau$. Then depth of $\tau$ can be calculated from the binary invariants by the following simple algorithm.

(1) Write the binary word $s_{2} s_{3} \cdots s_{n}$ as $O_{1} Z_{1} O_{2} Z_{2} \cdots O_{k} Z_{k}$, where $O_{i}$ and $Z_{i}$ are respectively maximal blocks of ones and zeros (thus $O_{1}$ and $Z_{k}$ may have length 0 , while all others have positive length).

(2) The depth of $\tau$ is $1+\sum_{j=1}^{k}\left\lceil\right.$ length $\left.\left(O_{i}\right) / 2\right\rceil$, where $\left\lceil\right.$ length $\left.\left(O_{i}\right) / 2\right\rceil$ denotes the least integer greater than or equal to length $\left(O_{i}\right) / 2$. 


\section{Heegaard diagrams of 3-manifolds}

In this section we will briefly review a method of Homma, Ochiai and Takahashi [9], and give some terminology.

Following [9] we will recall Heegaard diagrams for 3-manifolds. Let $H$ be a handlebody of genus-2.

Definition 3.1 A simple closed curve $u$ in $\partial H$ is called a meridian if there is a nonseparating disk $D$ in $H$ such that $D \cap \partial H=\partial D=u$. A set of simple closed curves $u_{1}, u_{2} \subset \partial H$ is a meridian system of $H$ if there are nonseparating disks $D_{1}, D_{2}$ bounded by $u_{1}, u_{2}$ such that $D_{1} \cap D_{2}=\varnothing$, and $\mathrm{Cl}\left(H-\mathrm{N}\left(D_{1} \cup D_{2}\right)\right)$ is homeomorphic to the 3-ball.

Remark 3.2 There are natural one-to-one correspondences between isotopy classes of meridians of $H$ and vertices of $\mathcal{D}(H)$, and between isotopy classes of meridian systems of $H$ and edges of $\mathcal{D}(H)$. So we say that a meridian or meridian system of $H$ is primitive if it corresponds to a primitive disk or pair respectively.

Let $\left\{u_{1}, u_{2}\right\}$ be a meridian system of $H$, and let $l$ be a simple arc in $\partial H$ such that $l$ intersects $u_{1}$ in $\partial l$, that both ends of $l$ attach on the same side of $u_{1}$, and that $l$ is disjoint from $u_{2}$. Then $u_{1}$ is cut into two arcs $\alpha^{\prime}$ and $\alpha^{\prime \prime}$ by $l$. Let $u_{1}^{\prime}=l \cup \alpha^{\prime}$ and $u_{1}^{\prime \prime}=l \cup \alpha^{\prime \prime}$. Then one of $\left\{u_{1}^{\prime}, u_{2}\right\}$ and $\left\{u_{1}^{\prime \prime}, u_{2}\right\}$ is a new meridian system of $H$.

Definition 3.3 This replacement is called an $l$-modification or modification along $l$.

Let $H \cup_{F} H^{\prime}$ be a genus-2 Heegaard splitting of a closed orientable 3-manifold $M$ and $\left\{u_{1}, u_{2}\right\}$ (resp. $\left\{v_{1}, v_{2}\right\}$ ) be a meridian system of $H$ (resp. $H^{\prime}$ ).

Definition 3.4 We call $D=\left(F ;\left\{u_{1}, u_{2}\right\},\left\{v_{1}, v_{2}\right\}\right)$ a genus-2 Heegaard diagram for $M$.

This definition extends to a definition for any genus Heegaard diagram of a nonclosed compact orientable 3-manifold (so that $H, H^{\prime}$ may be compression bodies), by choosing collections of core disks of 2-handles for each compression body $H, H^{\prime}$.

Remark 3.5 Let $\tau$ be a tunnel and $D$ a disk in the standard genus-2 handlebody $H$ corresponding to $\tau$. The exterior $\mathrm{E}\left(K_{\tau}\right)$ of $K_{\tau}$ is homeomorphic to $\mathrm{Cl}\left(S^{3}-H\right) \cup$ $(H \cap \mathrm{N}(D))$. Hence, by choosing a meridian system $\left\{v_{1}, v_{2}\right\}$ of $\mathrm{Cl}\left(S^{3}-H\right)$, we obtain a Heegaard diagram $\left(\partial H ; \partial D,\left\{v_{1}, v_{2}\right\}\right)$ for $\mathrm{E}\left(K_{\tau}\right)$. 
A Heegaard diagram $D=\left(F ;\left\{u_{1}, u_{2}\right\},\left\{v_{1}, v_{2}\right\}\right)$ is said to be normalized if $\left(u_{1} \cup u_{2}\right) \cap$ $\left(v_{1} \cup v_{2}\right)$ contains no isotopically removable points. For a non-normalized Heegaard diagram, an operation removing the isotopically removable intersection points by isotopy is called a normalization. When $\left(u_{1} \cup u_{2}\right) \cup\left(v_{1} \cup v_{2}\right)$ is connected, $D$ is called a connected diagram.

Definition 3.6 For a genus-2 normalized Heegaard diagram $D=\left(F ;\left\{u_{1}, u_{2}\right\},\left\{v_{1}, v_{2}\right\}\right)$, a simple arc $w$ in $F$ that satisfies the following conditions is called a $u_{i}$-wave or $\boldsymbol{u}$-wave (resp. $v_{i}$-wave or $\boldsymbol{v}$-wave) $(i \in\{1,2\})$, or just a wave.

- $w \cap\left(u_{1} \cup u_{2} \cup v_{1} \cup v_{2}\right)=w \cap u_{i}=\partial w$ (resp. $w \cap\left(u_{1} \cup u_{2} \cup v_{1} \cup v_{2}\right)=$ $\left.w \cap v_{i}=\partial w\right)$.

- Each component of $u_{i}-\partial w$ (resp. $\left.v_{i}-\partial w\right)$ intersects $v_{1} \cup v_{2}\left(\right.$ resp. $\left.u_{1} \cup u_{2}\right)$.

- At the two points $\partial w, w$ approaches the meridian $u_{i}$ (resp. $v_{i}$ ) from one side.

A modification along $w$ is called a wave transformation.

Remark 3.7 Let $D=\left(F\right.$; $\left.\left\{u_{1}, u_{2}\right\},\left\{v_{1}, v_{2}\right\}\right)$ be a genus-2 normalized Heegaard diagram for a 3-manifold $M$ and $w$ a $u_{1}$-wave. Then we can obtain a new Heegaard diagram $\left(F ;\left\{u_{1}^{\prime}, u_{2}\right\},\left\{v_{1}, v_{2}\right\}\right)$ for $M$. By a wave transformation, one of the components of $u_{1}-w$ disappears together with the intersection points of the meridians, and some more points may disappear under normalization. Hence the new diagram is always simpler than the old one in the sense that the total number of intersection points of the meridians decreases.

For a normalized Heegaard diagram, if there exists a wave, it can be taken so that the new diagram is also normalized. Throughout this paper, we assume that Heegaard diagrams are normalized and waves produce normalized Heegaard diagrams.

We consider genus-2 Heegaard diagrams $D=\left(F ;\left\{u_{1}, u_{2}\right\},\left\{v_{1}, v_{2}\right\}\right)$. By cutting $F$ open along $u_{1}$ and $u_{2}$ (or $v_{1}$ and $v_{2}$ ), we obtain the 2-sphere with four disks. We name these $A, a, B$ and $b$, where disks $A, a$ are obtained by cutting $F$ open along $u_{1}$ and disks $B, b$ are obtained by cutting $F$ open along $u_{2}$. Then by Ochiai [11], genus-2 normalized Heegaard diagrams are classified into type 1, 2, and 3 (as in Figure 1). In Figure 1, the labels on edges $c, d, e$, and $f$ indicate how many parallel edges there are in each parallel family of arcs.

Note that a nonconnected, genus-2 normalized Heegaard diagram for $S^{3}$ is the standard one $D_{0}=\left(F ;\left\{u_{1}, u_{2}\right\},\left\{v_{1}, v_{2}\right\}\right)$, where $u_{i}$ crosses $v_{j}$ at one point if $i=j$ and is disjoint from $v_{j}$ if $i \neq j$. 

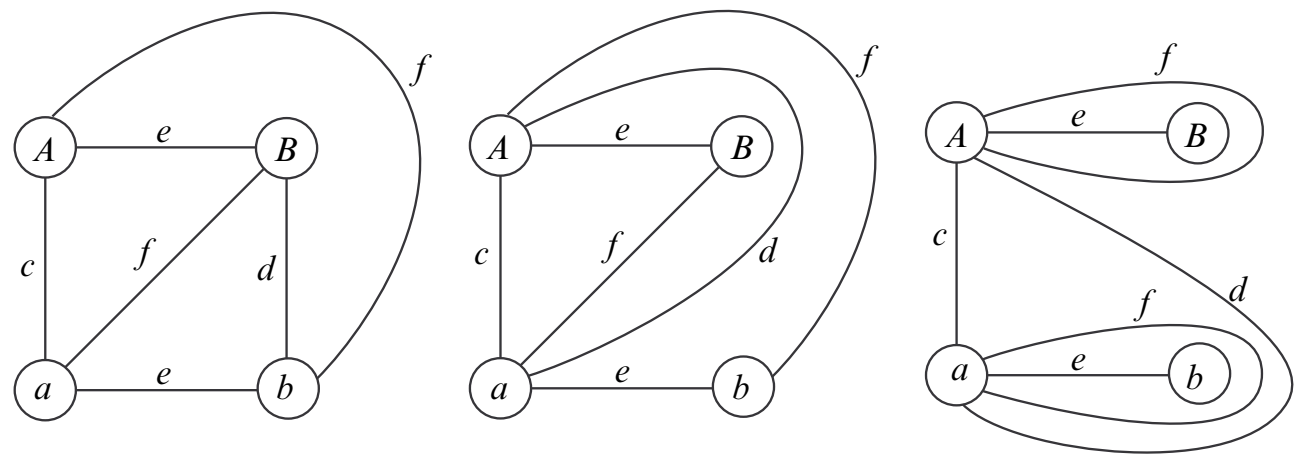

Figure 1: Types of genus-2 Heegaard diagram

Theorem 3.8 [9] Any connected normalized genus-2 Heegaard diagram for $S^{3}$ has a wave. Hence, through wave transformations any genus-2 Heegaard diagram for $S^{3}$ changes into the standard one.

For a survey of the proof, see Fomenko and Matveev [5].

\section{Link diagrams and Heegaard diagrams}

In this section we describe a relation between link diagrams with unknotting tunnels and genus-2 Heegaard diagrams for link exteriors.

First we show how to obtain a genus-2 Heegaard diagram for the exterior of a tunnel number one knot or link, from its link diagram with an unknotting tunnel. Let $K$ be a tunnel number one knot or link and $\alpha$ be an unknotting tunnel for $K$. We denote by $\mathrm{E}(K)$ the exterior $\mathrm{Cl}\left(S^{3}-\mathrm{N}(K)\right)$ of $K$. Put $h=\mathrm{N}(\alpha) \cap \mathrm{E}(K)$ and $H=\mathrm{N}(K) \cup h$. Note that $h$ is a 1 -handle for $\mathrm{N}(K)$ and a $2-$ handle for $\mathrm{Cl}\left(S^{3}-H\right)$. That is, $\mathrm{E}(K)$ is the union of a genus-2 handlebody $\mathrm{Cl}\left(S^{3}-H\right)$ and a 2-handle $h$ for $\mathrm{Cl}\left(S^{3}-H\right)$. The cocore disk $D$ of $h$ is an essential properly embedded disk in $H$. We have a genus-2 Heegaard splitting $H \cup \mathrm{Cl}\left(S^{3}-H\right)$ of $S^{3}$. Since $S^{3}$ has a unique Heegaard splitting of each genus, we may move $H$ by isotopy to the standard handlebody in $S^{3}$. Choosing a meridian system $\left\{v_{1}, v_{2}\right\}$ of $\mathrm{Cl}\left(S^{3}-H\right)$, we obtain a genus-2 Heegaard diagram $\left(\partial H ; \partial D,\left\{v_{1}, v_{2}\right\}\right)$ for $\mathrm{E}(K)$. In this way, each choice of a meridian system determines a genus- 2 Heegaard diagram for $\mathrm{E}(K)$. Figure 2 illustrates the process for a case where $K$ is the trefoil knot.

Next we show how to obtain a link diagram with an unknotting tunnel from a genus-2 Heegaard diagram for the exterior. Let $M$ be the exterior of a tunnel number one 


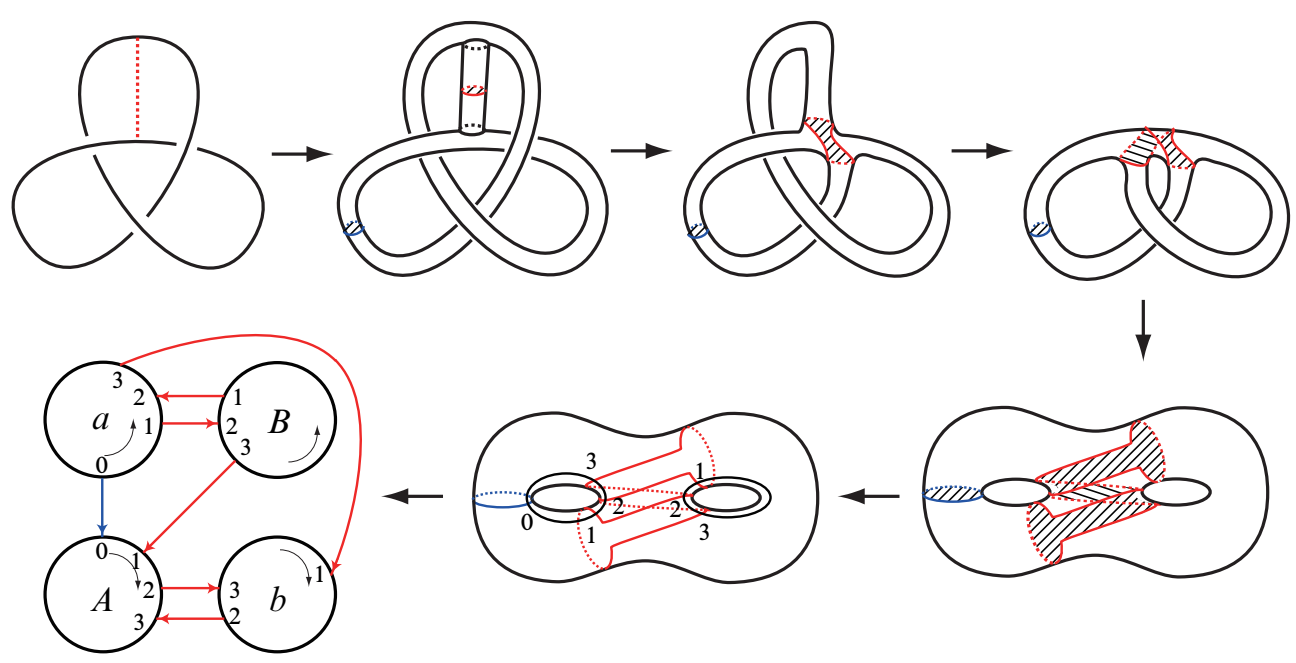

Figure 2: From a knot diagram to a Heegaard diagram

knot $K$ or two-component link $K=K_{1} \cup K_{2}$ in $S^{3}$, and let $\left(F ; u,\left\{v_{1}, v_{2}\right\}\right)$ be a genus-2 Heegaard diagram for $M$. Then there is an embedding $e: M \rightarrow S^{3}$ such that $e(M)=\mathrm{E}(K)$. The image of $F$ separates $S^{3}$ into two genus-2 handlebodies $H$ and $H^{\prime}$, where the image of $u$ bounds a disk in $H$ and each image of $v_{1}$ and $v_{2}$ bounds a disk in $H^{\prime}$. There exist simple closed curves $m_{1}, m_{2}$ in $F$ such that $\left\{e\left(m_{1}\right), e\left(m_{2}\right)\right\}$ is a meridian system of $H$ and $u \cap\left(m_{1} \cup m_{2}\right)=\varnothing$. We may assume that $m_{1}=u$ and $e\left(m_{2}\right)$ is a meridian of $K$ if $K$ is a knot, or that $e\left(m_{i}\right)$ is a meridian of $K_{i}$ for $i \in\{1,2\}$ if $K$ is a two-component link $K_{1} \cup K_{2}$. Then $\left(F ;\left\{m_{1}, m_{2}\right\},\left\{v_{1}, v_{2}\right\}\right)$ is a Heegaard diagram for $S^{3}$, and we will obtain a link diagram of $K$ from this. By Gordon and Luecke [8], the embedding $e$ is uniquely determined from $M$ if $K$ is a nontrivial knot. Berge [1] proved the following theorem.

Theorem 4.1 If $K$ is a nontrivial knot and $\left(F ; u,\left\{v_{1}, v_{2}\right\}\right)$ has neither a $v_{1}$-wave nor a $v_{2}$-wave, then there exists a $u$-wave that produces a simple closed curve $m$ in $F$ such that $e(m)$ is a meridian of $H$.

If $K$ is a two component link, $M$ has no essential annulus and $\left(F ; u,\left\{v_{1}, v_{2}\right\}\right)$ has neither a $v_{1}$-wave nor a $v_{2}$-wave, then there exists a $u$-wave that produces a simple closed curve $m$ in $F$ such that $e(m)$ is a meridian of $H$.

By Theorem 3.8, through a finite sequence of wave transformations we can change a Heegaard diagram $\left(F ;\left\{m_{1}, m_{2}\right\},\left\{v_{1}, v_{2}\right\}\right)$ to the standard one. Throughout these operations we will keep $u$ in mind. By changing a meridian system $\left\{m_{1}, m_{2}\right\}$ of $H$ 
(resp. $\left\{v_{1}, v_{2}\right\}$ of $H^{\prime}$ ) to a new one $\left\{u_{1}^{\prime}, u_{2}^{\prime}\right\}$ (resp. $\left\{v_{1}^{\prime}, v_{2}^{\prime}\right\}$ ), we can obtain the standard diagram $\left(F ;\left\{u_{1}^{\prime}, u_{2}^{\prime}\right\},\left\{v_{1}^{\prime}, v_{2}^{\prime}\right\}\right)$ without changing $u$. Then we regard $F$ as a standard genus-2 surface in $S^{3}$ and regard $H$ and $H^{\prime}$ as handlebodies in $S^{3}$. Moreover we move $H$ by isotopy so that $u$ becomes shorter and we can find a small disk $D$ in $H$ bounded by $u$. Then we obtain the knot corresponding to the embedding of $M$ by taking a core of the solid torus $\mathrm{Cl}(H-\mathrm{N}(D))$. Figure 3 illustrates the process for a case where $K$ is the trefoil knot.
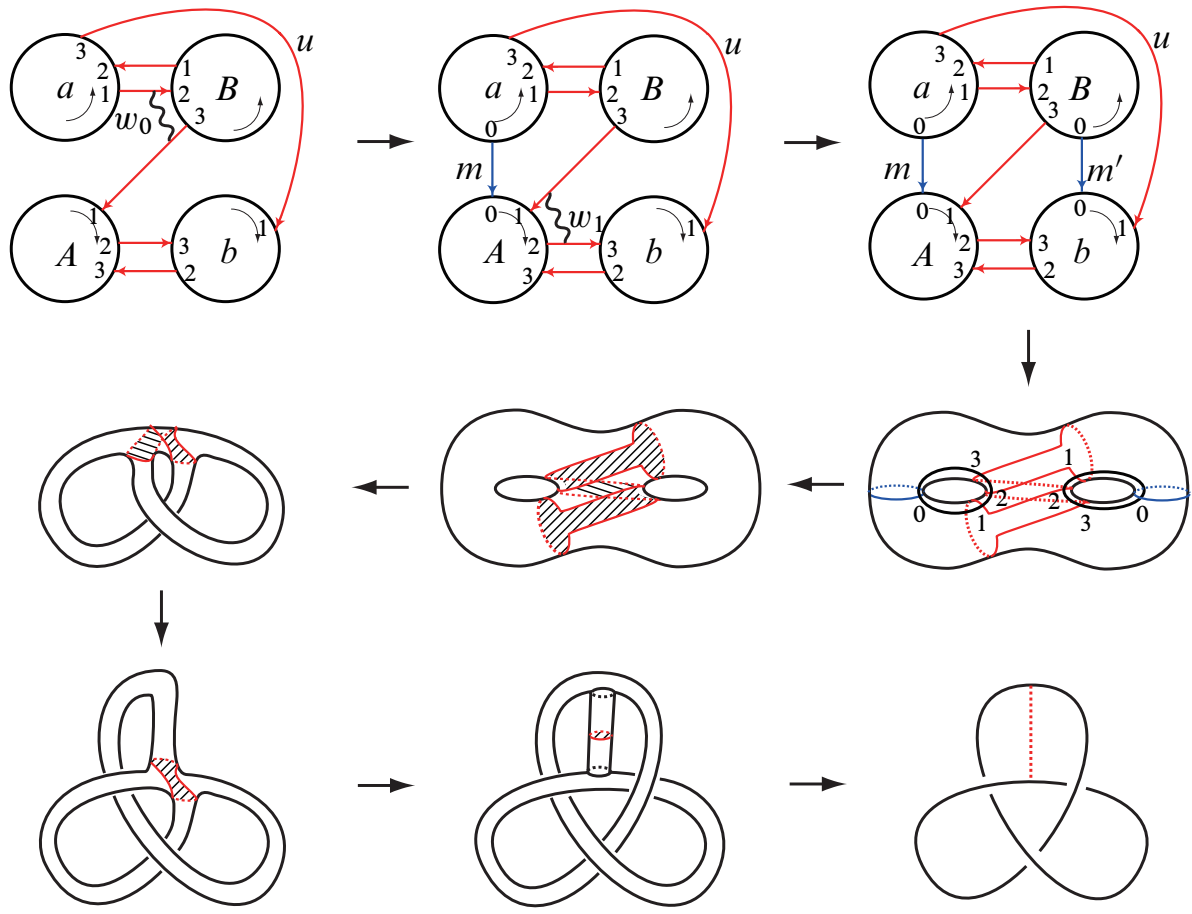

Figure 3: From a Heegaard diagram to a knot diagram

\section{Slope coordinates}

In this section we show how to calculate the rational invariant at each step of the principal path for the algorithm. Let $\mu=\{\lambda, \rho\}$ be a pair of nonseparating disks in $H, \sigma$ and $\sigma^{\prime}$ be slope disks for $\mu$. A part of $\mathcal{D}^{\prime}(H)$ is shown in Figure 4. We consider a diagram $\left(\partial H ;\{\partial \lambda, \partial \rho, \partial \sigma\},\left\{v_{1}, v_{2}\right\}\right)$, where $\left\{v_{1}, v_{2}\right\}$ is a meridian system of $\mathrm{Cl}\left(S^{3}-H\right)$. To calculate the $(\mu ; \sigma)$-slope of $\sigma^{\prime}$, we will find $\partial \sigma^{0}$ using a Heegaard diagram $\left(\partial H ;\{\partial \lambda, \partial \rho\},\left\{v_{1}, v_{2}\right\}\right)$. Here $\sigma^{0}$ is the slope 0 perpendicular disk for $(\mu ; \sigma)$. To 


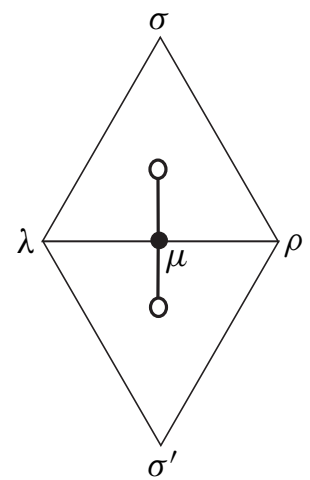

Figure 4: A part of $\mathcal{D}^{\prime}(H)$

find $\partial \sigma^{0}$, we give an orientation to each curve of $\partial \lambda, \partial \rho$, and $\partial \sigma$ so that each component of $\partial H-(\partial \lambda \cup \partial \rho \cup \partial \sigma)$ has an orientation induced by the orientation of $\partial \lambda \cup \partial \rho \cup \partial \sigma$ (see Figure 5). For an oriented simple closed curve $C^{\prime}$ in $\partial H$, by counting the algebraic

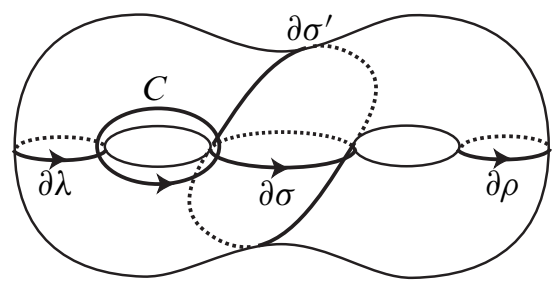

Figure 5: Curves on $\partial H$

intersection number with $v_{1}$ and $v_{2}$, we regard $C^{\prime}$ as an element of $H_{1}\left(\mathrm{Cl}\left(S^{3}-H\right)\right)$. We write $\left[C^{\prime}\right]$ for $\left(\triangle\left(C^{\prime}, v_{1}\right), \triangle\left(C^{\prime}, v_{2}\right)\right)$, where $\triangle$ means the algebraic intersection number on $\partial H$. We choose $C^{\prime}$ to intersect $\mu$ at one point, say $C^{\prime} \cap \mu=C^{\prime} \cap \partial \lambda=\{*\}$, and intersect $\partial \sigma$ at one point. We orient $C^{\prime}$ so that it meets $\partial \sigma$ (resp. $\partial \lambda$ ) from the right (resp. left) hand side. Since $S^{3}$ is obtained from the genus-2 handlebody $\mathrm{Cl}\left(S^{3}-H\right)$ by attaching 2 -handles along $\partial \sigma$ and $\partial \lambda$ and capping off a 3 -handle, the quotient $(\mathbb{Z} \oplus \mathbb{Z}) /\langle[\partial \sigma],[\partial \lambda]\rangle$ is isomorphic to $H_{1}\left(S^{3}\right)=0$. Therefore $[\partial \sigma]$ and $[\partial \lambda]$ form a basis of $\mathbb{Z} \oplus \mathbb{Z}$, and there are integers $k$ and $l$ such that $\left[C^{\prime}\right]=k[\partial \sigma]+l[\partial \lambda]$. Let $C=t_{\partial \sigma}^{-k}\left(C^{\prime}\right)$, where $t_{\alpha}$ denotes a right-handed Dehn twist along a curve $\alpha$ in $\partial H$.

Lemma 5.1 In $\partial H$, a boundary curve $e$ of a regular neighborhood of $\partial \lambda \cup C$ is isotopic to $\partial \sigma^{0}$.

Proof Let $E$ be a disk in $H$ bounded by $e$. Then $E$ is disjoint from $\lambda \cup \rho$, intersects $\sigma$ in an arc and separates $H$ into two solid tori. Let $K_{1}, K_{2}$ be core circles 
of $\mathrm{Cl}(H-\mathrm{N}(E))$. One of $K_{1}$ and $K_{2}$, say $K_{1}$, is isotopic to $C$ in $\mathrm{Cl}(H-\mathrm{N}(E))$. The curve $\partial \lambda$ is disjoint from $e$ and corresponds to a meridian of $K_{1}$, so $K_{1}$ is also isotopic to the curve $t_{\partial \lambda}^{l}(C)$ in $\mathrm{Cl}(H-\mathrm{N}(E))$. By the definition of $C,\left[t_{\partial \lambda}^{l}(C)\right]=$ $[C]-l[\partial \lambda]=\left[C^{\prime}\right]-k[\partial \sigma]-l[\partial \lambda]=0$ in $H_{1}\left(\mathrm{Cl}\left(S^{3}-H\right)\right)$. Then there is a Seifert surface of $K_{1}$ that is disjoint from $K_{2}$, and so the link $K_{1} \cup K_{2}$ has linking number 0 . By the definition of the slope-0 perpendicular disk (see Definition 2.5), $E$ is isotopic to $\sigma^{0}$ in $H$, and so $e$ is isotopic to $\partial \sigma^{0}$ in $\partial H$.

\section{The algorithm}

In this section we present the algorithm of Theorem 1.1.

Let $H$ be a standard unknotted genus-2 handlebody in $S^{3}$ and $\tilde{\tau}$ a disk in $H$, which represents the tunnel $\tau$ whose parameter we wish to find. Put $u=\partial \tilde{\tau}$ and let $\left\{m_{1}, m_{2}\right\}$ be a meridian system of $H$ that is disjoint from $u$. If $\tau$ is a knot tunnel, we choose $m_{1}=u$ and $m_{2}$ to correspond to the meridian of $K_{\tau}$ in the solid torus $\mathrm{Cl}(H-\mathrm{N}(\tilde{\tau}))$. Note that there are many choices of the meridian of $K_{\tau}$ in $\partial H$, but we choose one. If $\tau$ is a link tunnel, we may assume that each of $m_{1}$ and $m_{2}$ corresponds to the meridian in $\mathrm{Cl}(H-\mathrm{N}(\tilde{\tau}))$ of each knot component of $K_{\tau}$. Let $\boldsymbol{v}$ be some meridian system of the genus -2 handlebody $\mathrm{Cl}\left(S^{3}-H\right)$. We will find the slope coordinates one by one, while finding the sequence $\boldsymbol{\alpha}_{\mathbf{0}}, \ldots, \boldsymbol{\alpha}_{\boldsymbol{n}}$ of meridian systems of $H$ that corresponds to the principal path of $\tau$.

Step 0 We will find $\boldsymbol{\alpha}_{\mathbf{0}}$ corresponding to the principal pair of $\tau$. If $\tau$ is a link tunnel, we may put $\boldsymbol{\alpha}_{\mathbf{0}}=\left\{m_{1}, m_{2}\right\}$ and skip Step 0. If $\tau$ is a knot tunnel, we put $\boldsymbol{\alpha}_{-\mathbf{1}}=\left\{m_{1}, m_{2}\right\}=\{u, m\}$. Although the choice of $m$ is not unique, $\boldsymbol{\alpha}_{\mathbf{0}}$ will be uniquely determined by Step 0 . We will obtain $\boldsymbol{\alpha}_{\mathbf{0}}$ from the Heegaard diagram $D_{-1}=\left(\partial H ; \boldsymbol{\alpha}_{-\mathbf{1}}, \boldsymbol{v}\right)$ for $S^{3}$ as follows.

$(0-1)$ Judge whether $D_{-1}$ is connected. If $D_{-1}$ is not connected, so is the standard diagram and $\alpha_{-1}$ is primitive. Hence $\tau$ is the unique tunnel of the trivial knot, the parameter is trivial, and we stop. If $D_{-1}$ is connected, go to (0-2).

(0-2) Judge whether $D_{-1}$ has a $\boldsymbol{v}$-wave. If $D_{-1}$ has a $\boldsymbol{v}$-wave, perform the wave transformation and go back to $(0-1)$ after replacing $v$ with a new one. If $D_{-1}$ has no $\boldsymbol{v}$-wave, go to (0-3).

(0-3) Judge whether $D_{-1}$ has an $m$-wave. If $D_{-1}$ has an $m$-wave, perform the wave transformation and go back to (0-1) after replacing $m$ with a new one. If $D_{-1}$ has no $m$-wave, go to (0-4).

(0-4) Then $D_{-1}$ must have a $u$-wave, and we perform the wave transformation. Let $\boldsymbol{\alpha}_{\mathbf{0}}$ be the meridian system of $H$ obtained by the wave transformation. 
In this way, we obtain $\boldsymbol{\alpha}_{\mathbf{0}}$ whenever $\tau$ is not the unique tunnel of the trivial knot, and we put $D_{0}=\left(\partial H ; \boldsymbol{\alpha}_{\mathbf{0}}, \boldsymbol{v}\right)$. For a positive integer $i$, we will define $D_{i}=\left(\partial H ; \boldsymbol{\alpha}_{\boldsymbol{i}}, \boldsymbol{v}\right)$ from a Heegaard diagram $D_{i-1}=\left(\partial H ; \boldsymbol{\alpha}_{\boldsymbol{i}-\mathbf{1}}, \boldsymbol{v}\right)$, and find the slope as follows.

\section{Step i}

( $i-1)$ Judge whether $D_{i-1}$ is connected. If $D_{i-1}$ is not connected, it is the standard diagram and we go to the Final Step below after putting $n=i-1$. If $D_{i-1}$ is connected, go to $(i-2)$.

(i-2) Judge whether $D_{i-1}$ has a $\boldsymbol{v}$-wave. If $D_{i-1}$ has a $\boldsymbol{v}$-wave, perform the wave transformation and go back to $(i-1)$ after replacing $\boldsymbol{v}$ with a new one. If $D_{i-1}$ has no $\boldsymbol{v}$-wave, go to $(i-3)$.

( $i-3) \quad D_{i-1}$ must have $\boldsymbol{\alpha}_{\boldsymbol{i}-\mathbf{1}}$-wave, and we perform the wave transformation. Let $\boldsymbol{\alpha}_{\boldsymbol{i}}$ be the meridian system of $H$ produced by the wave transformation and put $D_{i}=\left(\partial H ; \boldsymbol{\alpha}_{\boldsymbol{i}}, \boldsymbol{v}\right)$. Go to $(i-4)$.

( $i-4)$ Calculate the slope $r_{i-1}$. Following the notation of Section 5, regard $\{\partial \lambda, \partial \rho\}$ as $\boldsymbol{\alpha}_{\boldsymbol{i}-\mathbf{1}},\left\{\partial \sigma^{\prime}\right\}$ as $\boldsymbol{\alpha}_{\boldsymbol{i - 2}}-\boldsymbol{\alpha}_{\boldsymbol{i - 1}}$, and $\{\partial \sigma\}$ as $\boldsymbol{\alpha}_{\boldsymbol{i}}-\boldsymbol{\alpha}_{\boldsymbol{i}-\mathbf{1}}$. Calculate the $(\mu ; \sigma)-$ slope of $\sigma^{\prime}$, and write it as $r_{i-1}$. Go to Step 2 if $i=1$, otherwise go to (i-5).

( $i-5)$ Determine the binary number $t_{i-2}$ by putting $t_{i-2}=0$ or $t_{i-2}=1$ according to whether or not the unique meridian $\boldsymbol{\alpha}_{\boldsymbol{i - 2}} \cap \boldsymbol{\alpha}_{\boldsymbol{i - 1}}$ equals the unique meridian $\boldsymbol{\alpha}_{\boldsymbol{i}-\mathbf{1}} \cap \boldsymbol{\alpha}_{\boldsymbol{i}}$. Then go to Step $i+1$.

Continue these operations inductively until a standard diagram $D_{n}=\left(\partial H ; \boldsymbol{\alpha}_{\boldsymbol{n}}, \boldsymbol{v}\right)$ is obtained. By Remark 3.7, we will reach the Final Step below after finitely many steps.

Final Step Calculate the slope $r_{n}$. Let $\alpha$ be a simple closed curve in $\partial H$ that is disjoint from $\boldsymbol{\alpha}_{\boldsymbol{n}}$ and intersects each $v_{i}$ of $\boldsymbol{v}$ at one point. Regarding $\{\partial \lambda, \partial \rho\}$ as $\boldsymbol{\alpha}_{\boldsymbol{n}}$, $\left\{\partial \sigma^{\prime}\right\}$ as $\boldsymbol{\alpha}_{\boldsymbol{n}-\boldsymbol{1}}-\boldsymbol{\alpha}_{\boldsymbol{n}}$, and $\partial \sigma$ as $\alpha$, we calculate the $(\mu ; \sigma)$-slope of $\sigma^{\prime}$, and write it as $r_{n}$. Then stop. Note that the choice of $\alpha$ is not unique, but $\left[1 / r_{n}\right]$ is uniquely determined.

Theorem 6.1 The parameter of $\tau$ is $\left(\left(\left[1 / r_{n}\right], r_{n-1}, \ldots, r_{0}\right),\left(t_{n-2}, \ldots, t_{0}\right)\right)$.

Theorem 6.1 and consequently Theorem 1.1 follow immediately from the next result. For each integer $i$ satisfying $0 \leq i \leq n$, let $\tilde{\mu}_{i}$ be a pair of disks in $H$ corresponding to $\alpha_{n-i}$ and let $\mu_{i}$ be the $\mathcal{G}$-orbit of $\tilde{\mu}_{i}$.

Theorem 6.2 The path $\theta_{0}, \mu_{0}, \mu_{0} \cup \mu_{1}, \mu_{1}, \ldots, \mu_{n}, \mu_{n} \cup\{\tau\}$ defined above is the principal path of $\tau$.

The proof of Theorem 6.2 is given the next section. 


\section{Proof of Theorem 6.2}

The proof of Theorem 6.2 will use Lemmas 7.1 and 7.2 below. Let $D=\left(\partial H ;\left\{u_{1}, u_{2}\right\}\right.$, $\left.\left\{v_{1}, v_{2}\right\}\right)$ be a genus-2 Heegaard diagram for $S^{3}$, ie $\left\{u_{1}, u_{2}\right\}$ is a meridian system of the standard genus-2 handlebody $H$ in $S^{3}$ and $\left\{v_{1}, v_{2}\right\}$ is a meridian system of the genus-2 handlebody $\mathrm{Cl}\left(S^{3}-H\right)$.

Lemma 7.1 Suppose that $D$ has a $u_{1}$-wave. Let $\left\{u_{1}^{\prime}, u_{2}\right\}$ be a new meridian system of $H$ obtained by a wave transformations along the $u_{1}$-wave and $D^{\prime \prime}=$ $\left(F ;\left\{u_{1}^{\prime}, u_{2}\right\},\left\{v_{1}^{\prime}, v_{2}^{\prime}\right\}\right)$ a Heegaard diagram obtained from $D^{\prime}=\left(F ;\left\{u_{1}^{\prime}, u_{2}\right\},\left\{v_{1}, v_{2}\right\}\right)$ by wave transformations along waves approaching meridians of $\mathrm{Cl}\left(S^{3}-H\right)$. Then $u_{1}$ cannot be obtained from $\left(F ;\left\{u_{1}^{\prime}, u_{2}\right\},\left\{v_{1}^{\prime}, v_{2}^{\prime}\right\}\right)$ by a wave transformation along a $u_{1}^{\prime}$-wave or $u_{2}$-wave.

This lemma implies that $\boldsymbol{\alpha}_{\boldsymbol{i}-\mathbf{1}} \cup \boldsymbol{\alpha}_{\boldsymbol{i}}$ and $\boldsymbol{\alpha}_{\boldsymbol{i}} \cup \boldsymbol{\alpha}_{\boldsymbol{i}+\mathbf{1}}$ in the algorithm correspond to different vertices in $\tilde{\mathcal{T}}$ for $0 \leq i \leq n-1$.

Proof of Lemma 7.1 In this proof we regard Heegaard diagrams as the 2-spheres $S$ with four disks obtained by cutting $\partial H$ open along $u_{1}^{\prime}$ and $u_{2}$. The union $v_{1} \cup v_{2}$ or $v_{1}^{\prime} \cup v_{2}^{\prime}$ splits into families of parallel edges. The key to the proof is a symmetry for any genus -2 normalized Heegaard diagram: the pairs of families of parallel edges labeled $e$ or $f$ in Figure 1 have the same numbers of elements.

First we consider a wave approaching a meridian of $\mathrm{Cl}\left(S^{3}-H\right)$ that produces a normalized Heegaard diagram, and show that the number of parallel edges joining $u_{1}^{\prime}$ and $u_{2}$ does not increase under such a wave transformation. Each edge of the new meridian is an edge of the old meridian, except an edge $\widehat{w}$ that contains the wave. Thus the number of parallel edges does not increase, except the number of edges parallel to $\widehat{w}$. For the pairs of families containing an equal number of edges, one of the families is not parallel to $\widehat{w}$, so its number does not increase. By the symmetry condition, neither does the other. Consequently the number of parallel edges joining $u_{1}^{\prime}$ and $u_{2}$ does not increase.

Next we consider a $u_{1}$-wave $w_{1}$ that produces $u_{1}^{\prime}$, and show that $D^{\prime \prime}$ has no edge of $v_{1}^{\prime} \cup v_{2}^{\prime}$ that joins $u_{1}^{\prime}$ and $u_{2}$ and is disjoint from $u_{1}$. Since $u_{1}^{\prime}$ is obtained from $u_{1}$ by a wave transformation along $w_{1}, u_{1}$ and $w_{1}$ can be moved slightly so that both are disjoint from $u_{1}^{\prime} \cup u_{2}$, so lie on $S$ as a loop and an arc respectively. Each component obtained from $S$ by cutting open along $u_{1}$ contains exactly two disks, one corresponding to $u_{1}^{\prime}$ and the other to $u_{2}$. Let $F$ be the component that contains $w_{1}$. The $u_{1}$-wave $w_{1}$ separates two disks in $F$ and is disjoint from $v_{1} \cup v_{2}$, so $F$ contains no edge of $v_{1} \cup v_{2}$ joining $u_{1}^{\prime}$ and $u_{2}$. By the symmetry condition, $\mathrm{Cl}(S-F)$ also 
contains no edge of $v_{1} \cup v_{2}$ joining $u_{1}^{\prime}$ and $u_{2}$. Thus $D^{\prime}$ has no edge of $v_{1} \cup v_{2}$ that joins $u_{1}^{\prime}$ and $u_{2}$ and disjoint from $u_{1}$. Since $D^{\prime \prime}$ is obtained from $D^{\prime}$ by wave transformations along waves approaching meridians of $\mathrm{Cl}\left(S^{3}-H\right), D^{\prime \prime}$ also has no edge of $v_{1}^{\prime} \cup v_{2}^{\prime}$ that joins $u_{1}^{\prime}$ and $u_{2}$ and is disjoint from $u_{1}$.

Finally we suppose that $D^{\prime \prime}$ has a $u_{1}^{\prime}$-wave or $u_{2}$-wave, say a $u_{1}^{\prime}$-wave $w_{1}^{\prime}$, which produces $u_{1}$, and obtain a contradiction. By moving edges of $v_{1}^{\prime} \cup v_{2}^{\prime}$ if necessary, we may assume that $u_{1} \cap\left(v_{1}^{\prime} \cup v_{2}^{\prime}\right)$ has no isotopically removable points. We can move $w_{1}^{\prime}$ by isotopy so that it is disjoint from $u_{1}$ and still disjoint from $v_{1}^{\prime} \cup v_{2}^{\prime}$. Let $F^{\prime}$ be the component obtained from $S$ by cutting open along $u_{1}$ that contains $w_{1}^{\prime}$, and let $D_{1}^{\prime}$ and $D_{2}$ be disks in $F^{\prime}$ corresponding to $u_{1}^{\prime}, u_{2}$ respectively. Then $w_{1}^{\prime} \cap\left(D_{1}^{\prime} \cup D_{2}\right)=w_{1}^{\prime} \cap \partial D_{1}^{\prime}=\partial w_{1}^{\prime}$, and $\partial F^{\prime}$ and $D_{2}$ are contained in different components of $F^{\prime}-\left(D_{1}^{\prime} \cup w_{1}^{\prime}\right)$. Let $F^{\prime \prime}$ be the closure of the component of $F^{\prime}-\left(D_{1}^{\prime} \cup w_{1}^{\prime}\right)$ that contains $D_{2}$. Now $\partial F^{\prime \prime}$ consists of $w_{1}^{\prime}$ and a subarc $\alpha$ of $\partial D_{1}^{\prime}$. Since $F^{\prime \prime}$ (and also $F^{\prime}$ ) contains no edge of $v_{1}^{\prime} \cup v_{2}^{\prime}$ joining $\alpha$ and $\partial D_{2}$, and $w_{1}^{\prime}$ is disjoint from $v_{1}^{\prime} \cup v_{2}^{\prime}, F^{\prime \prime}$ contains no edge of $v_{1}^{\prime} \cup v_{2}^{\prime}$. This implies that $u_{2}$ is disjoint from $v_{1}^{\prime} \cup v_{2}^{\prime}$. Consequently $u_{1}^{\prime} \cup u_{2} \cup v_{1}^{\prime} \cup v_{2}^{\prime}$ is not connected, ie $D^{\prime \prime}$ is not connected. Any nonconnected genus -2 normalized Heegaard diagram for $S^{3}$ is standard, so each meridian intersects another one at one point. Hence $D^{\prime \prime}$ is neither connected nor standard. This contradicts the fact that $D^{\prime \prime}$ is a Heegaard diagram for $S^{3}$.

Lemma 7.2 Suppose that $\left\{u_{1}, u_{2}\right\}$ is primitive and $D$ is connected. Then $D$ has a $v_{1}$-wave or $v_{2}$-wave.

This lemma implies that $\boldsymbol{\alpha}_{\boldsymbol{i}}$ in the algorithm is not primitive for $0 \leq i \leq n-1$.

Proof of Lemma 7.2 Let $D_{1}$ and $D_{2}$ be disks in $\mathrm{Cl}\left(S^{3}-H\right)$ bounded by $v_{1}$ and $v_{2}$ respectively. Since $\left\{u_{1}, u_{2}\right\}$ is primitive, there exists a $2-$ sphere that intersects $H$ in a disk and splits $u_{1}$ from $u_{2}$. Let $S$ be such a 2 -sphere that has a minimal intersection number $\left|S \cap\left(D_{1} \cup D_{2}\right)\right|$. Now $D_{1} \cup D_{2}$ is not disjoint from $S$, since otherwise $D=\left(\partial H ;\left\{u_{1}, u_{2}\right\},\left\{v_{1}, v_{2}\right\}\right)$ would be nonconnected. Consider an outermost disk in $S$. Its boundary consists two $\operatorname{arcs} w$ and $w^{\prime}$ that are a part of $S \cap \partial H$ and a part of $S \cap\left(D_{1} \cup D_{2}\right)$ respectively. Then $w$ is a $v_{1}$-wave or $v_{2}$-wave.

Proof of Theorem 6.2 Let $\widetilde{\mathcal{T}_{0}}$ be the primitive subtree, that is the subcomplex of $\widetilde{\mathcal{T}}$ spanned by the vertices that are primitive pairs and primitive triple. Since $D_{n}=$ $\left(\partial H ; \boldsymbol{\alpha}_{\boldsymbol{n}}, \boldsymbol{v}\right)$ is standard, $\tilde{\mu}_{0}$ is primitive and $\tilde{\mu}_{n}, \tilde{\mu}_{n} \cup \tilde{\mu}_{n-1}, \tilde{\mu}_{n-1}, \ldots, \tilde{\mu}_{0}$ is a path in $\tilde{\mathcal{T}}$ from $\tilde{\mu}_{n}$ to $\widetilde{\mathcal{T}}_{0}$. Lemma 7.1 and Lemma 7.2 show that the path is the shortest path in $\tilde{\mathcal{T}}$ from $\tilde{\mu}_{n}$ to $\widetilde{\mathcal{T}_{0}}$. The action of $\mathcal{G}$ leaves $\widetilde{\mathcal{T}}_{0}$ invariant, hence preserve the shortest path. Consequently the path $\mu_{n}, \mu_{n} \cup \mu_{n-1}, \mu_{n-1}, \ldots, \mu_{0}$ is the unique shortest path from $\mu_{n}$ to $\mu_{0}$, so $\theta_{0}, \mu_{0}, \mu_{0} \cup \mu_{1}, \mu_{1}, \ldots, \mu_{n}, \mu_{n} \cup\{\tau\}$ is the principal path of $\tau$. 


\section{Example}

In [7, Section 10], Goda and Hayashi called attention to the tunnels $\gamma_{1}$ and $\gamma_{2}$ of the twisted torus knot $T(5,7,2)$, illustrated in Figure 6 . A preliminary version [6] of their paper had a gap: They had asserted that the tunnel $\gamma_{1}$ is a $(1,1)-$ tunnel and $\gamma_{2}$ is a non- $(1,1)-$ tunnel because of $[10,(1.3)$ Proposition]. According to that Proposition, when a tunnel is represented by a tunnel arc, the tunnel is $(1,1)$ if and only if one of the associated knots (an associated knot means a knot consisting of the tunnel arc and one of the two arcs into which the endpoints of the tunnel arc cut the knot) is trivial. In fact, when a tunnel is represented as an arc attached to a knot, the tunnel is $(1,1)$ if and only if the arc can be slid along the knot, with the endpoints possibly passing through each other on the knot, until one of the two associated knots is trivial. Since one of the associated knots of $\gamma_{1}$ is trivial, it does follow that $\gamma_{1}$ is $(1,1)$. For $\gamma_{2}$, neither associated knot is trivial, but there remains the possibility that $\gamma_{2}$ could be slid along the knot to make one of the associated knots trivial. In this section, we apply the algorithm to these tunnels and confirm that $\gamma_{1}$ is $(1,1)$ but $\gamma_{2}$ is not. This is the first example, other than torus knots, of a knot having both a $(1,1)-$ tunnel and a non-(1, 1)-tunnel.

By Remark 2.9, we can determine whether or not a given tunnel is $(1,1)$ by computing its depth, and by Proposition 2.10, the depth can be computed from the binary invariants $s_{2}, s_{3}, \ldots, s_{n}$. For this reason, in the application of the algorithm below, we will omit the calculation of the rational invariants, part (i-4) in each Step $i$ for $i \geq 1$, and in the Section 6.

Let $K$ be the twisted torus knot $T(5,7,2)$ and let $\gamma_{1}, \gamma_{2}$ be the tunnels illustrated in Figure 6. Below we will apply the algorithm presented in Section 6 to $\gamma_{1}$ and $\gamma_{2}$.

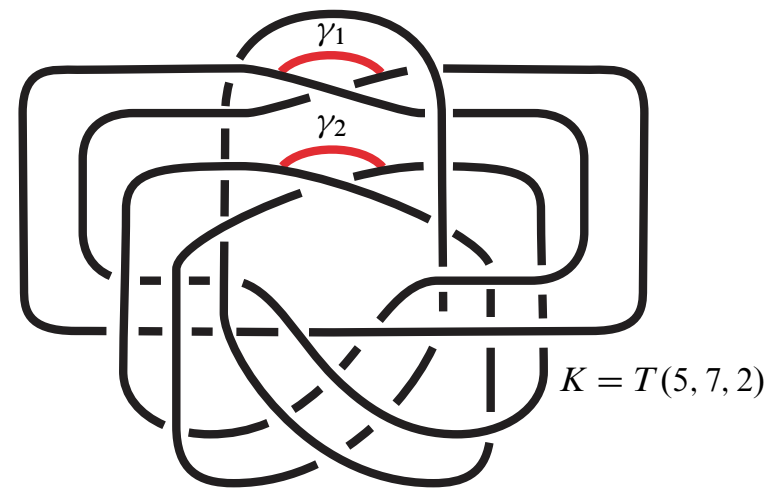

Figure 6: The example of Goda and Hayashi, 


\subsection{The tunnel $\gamma_{1}$}

A regular neighborhood $\mathrm{N}\left(K \cup \gamma_{1}\right)$ of $K \cup \gamma_{1}$ is an unknotted genus-2 handlebody, so it can be moved to the standard genus-2 handlebody $H$ in $S^{3}$ by isotopy as illustrated in Figure 7. Let $u_{1}$ be the meridian of $H$ that corresponds to $\gamma_{1}$ and $m_{1}$ a meridian of $H$
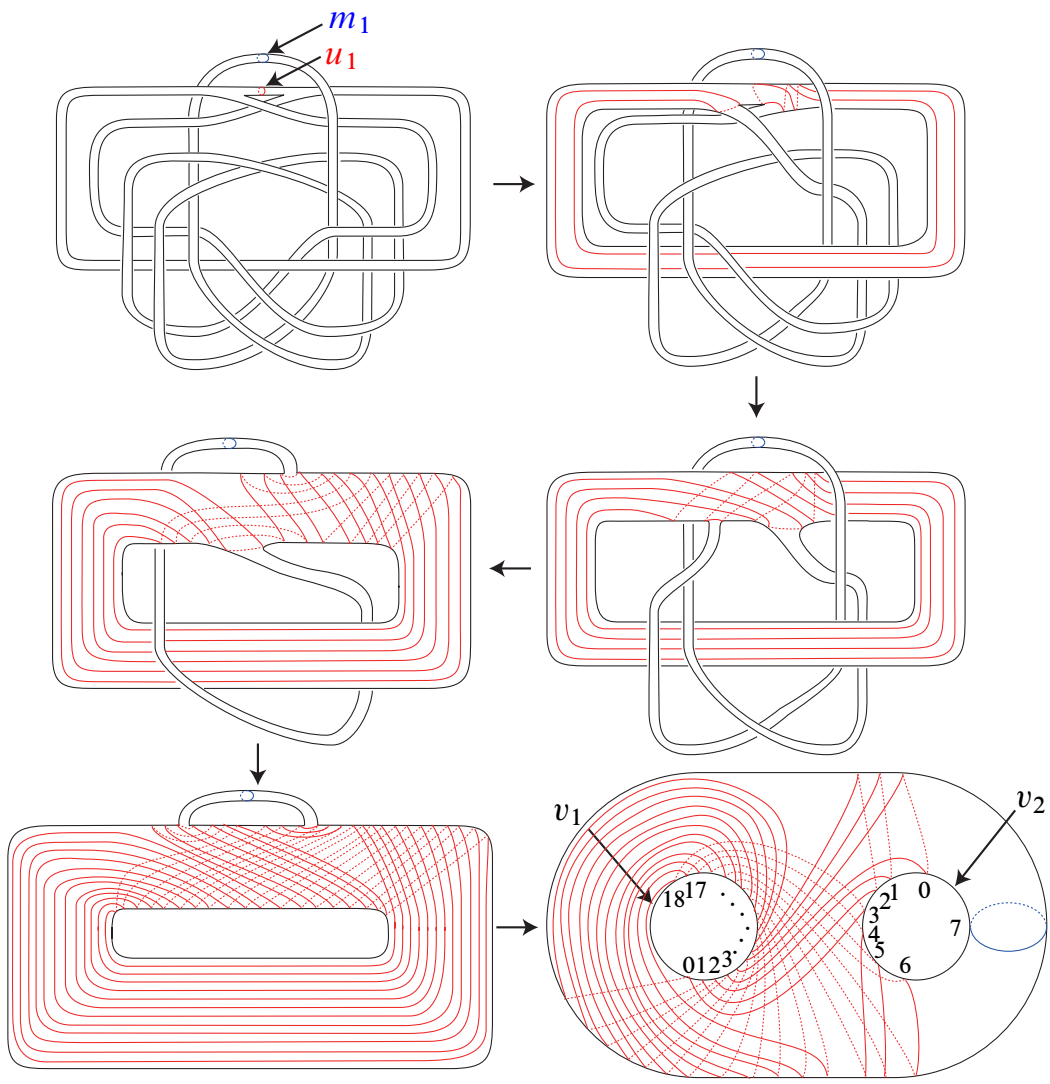

Figure 7: The tunnel $\gamma_{1}$

that corresponds to the meridian of $K$; see Figure 7. Cutting $\partial H$ open along meridians $v_{1}$ and $v_{2}$ of $\mathrm{Cl}\left(S^{3}-H\right)$, we obtain a Heegaard diagram $D_{-1}=\left(\partial H ; \boldsymbol{\alpha}_{-\mathbf{1}}, \boldsymbol{v}\right)$ for $S^{3}$ as illustrated in the left-hand side of Figure 8, where $\boldsymbol{\alpha}_{-\mathbf{1}}=\left\{u_{1}, m_{1}\right\}$ and $\boldsymbol{v}=\left\{v_{1}, v_{2}\right\}$. Now we apply the algorithm to $\gamma_{1}$ by using $D_{-1}$.

\section{Step 0}

(0-1) $\quad D_{-1}$ is connected, so we go to $(0-2)$.

(0-2) $D_{-1}$ has no $\boldsymbol{v}$-wave, so we go to (0-3). 

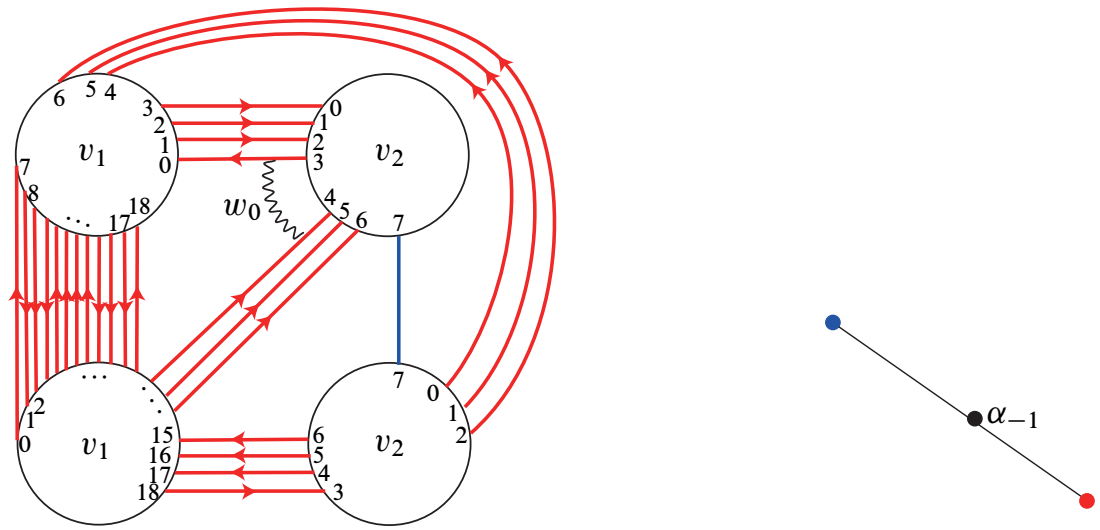

Figure 8: $D_{-1}=\left(\partial H ; \boldsymbol{\alpha}_{-\mathbf{1}}, \boldsymbol{v}\right)$

(0-3) $D_{-1}$ has no $m_{1}$-wave, so we go to (0-4).

(0-4) Then $D_{-1}$ has a $u_{1}$-wave $w_{0}$ as illustrated in Figure 8. A Heegaard diagram $D_{0}=\left(\partial H ; \boldsymbol{\alpha}_{\mathbf{0}}, \boldsymbol{v}\right)$ obtained by performing the wave transformation along $w_{0}$ is illustrated in Figure 9, and we go to Step 1 below.

\section{Step 1}

(1-1) $\quad D_{0}$ is connected, so we go to (1-2).

(1-2) $D_{0}$ has no $\boldsymbol{v}$-wave, so we go to (1-3).
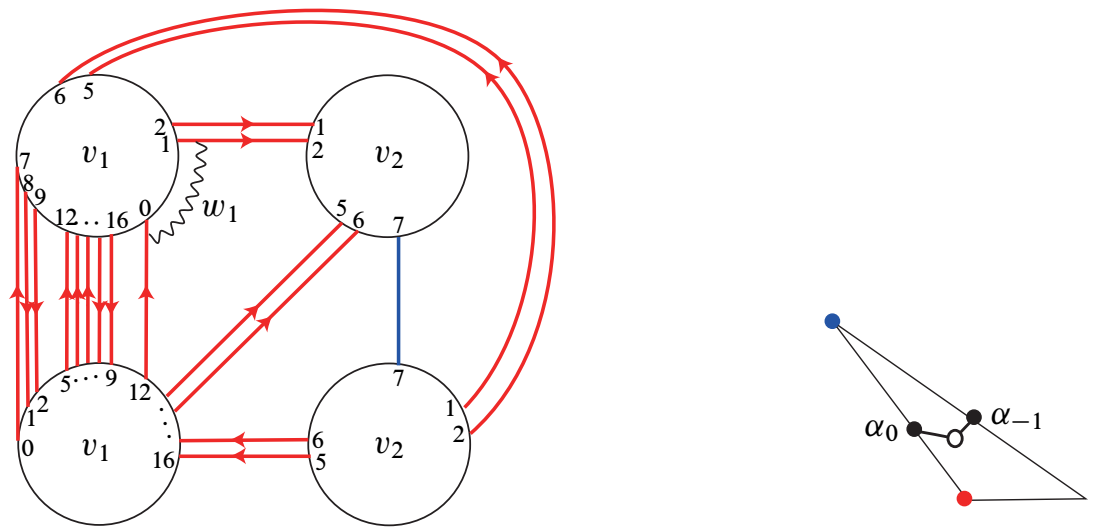

Figure 9: $D_{0}=\left(\partial H ; \boldsymbol{\alpha}_{\mathbf{0}}, \boldsymbol{v}\right)$ 
(1-3) Then $D_{0}$ has a $\boldsymbol{\alpha}_{\mathbf{0}}$-wave $w_{1}$ as illustrated in Figure 9. A Heegaard diagram $D_{1}=\left(\partial H ; \boldsymbol{\alpha}_{\mathbf{1}}, \boldsymbol{v}\right)$ obtained by performing the wave transformation along $w_{1}$ is illustrated in Figure 10, and we go to Step 2 below.
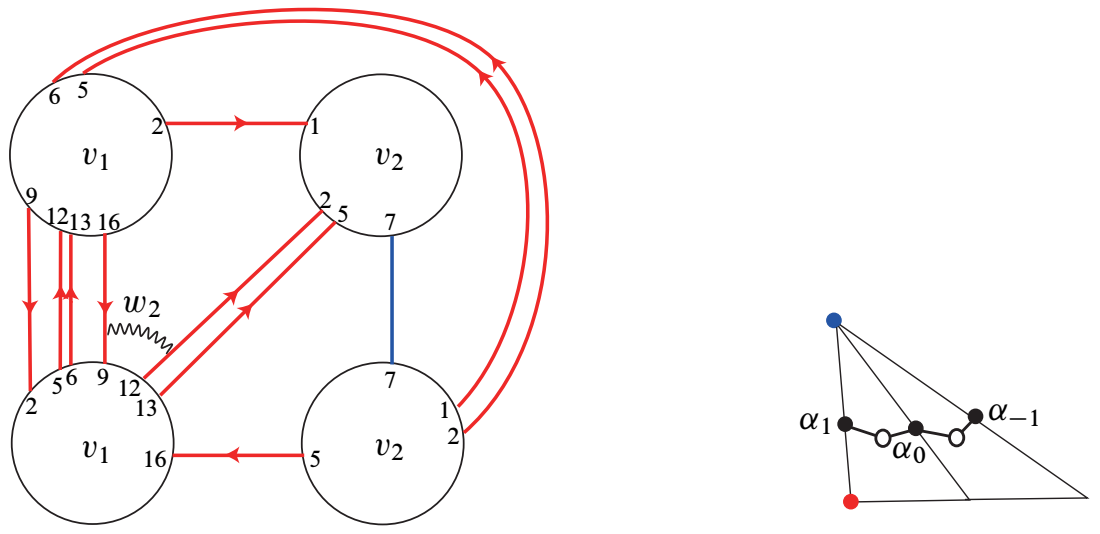

Figure 10: $D_{1}=\left(\partial H ; \boldsymbol{\alpha}_{\mathbf{1}}, \boldsymbol{v}\right)$

\section{Step 2}

(2-1) $\quad D_{1}$ is connected, so we go to (2-2).

(2-2) $D_{1}$ has no $\boldsymbol{v}$-wave, so we go to (2-3).

(2-3) Then $D_{1}$ has a $\boldsymbol{\alpha}_{1}$-wave $w_{2}$ as illustrated in Figure 10. A Heegaard diagram $D_{2}=\left(\partial H ; \boldsymbol{\alpha}_{\mathbf{2}}, \boldsymbol{v}\right)$ obtained by performing the wave transformation along $w_{2}$ is illustrated in Figure 11, and we go to (2-5).

(2-5) Put $t_{0}=0$ because the unique meridian $\alpha_{0} \cap \alpha_{1}$ equals the unique meridian $\boldsymbol{\alpha}_{\mathbf{1}} \cap \boldsymbol{\alpha}_{\mathbf{2}}$. Then go to Step 3 below.

\section{Step 3}

(3-1) $D_{2}$ is connected, so we go to (3-2).

(3-2) $D_{2}$ has no $\boldsymbol{v}$-wave, so we go to (3-3).

(3-3) Then $D_{2}$ has a $\boldsymbol{\alpha}_{2}$-wave $w_{3}$ as illustrated in Figure 11. A Heegaard diagram $D_{3}=\left(\partial H ; \boldsymbol{\alpha}_{\mathbf{3}}, \boldsymbol{v}\right)$ obtained by performing the wave transformation along $w_{3}$ is illustrated in Figure 12, and we go to (3-5).

(3-5) Put $t_{0}=0$ because the unique meridian $\alpha_{1} \cap \alpha_{2}$ equals the unique meridian $\boldsymbol{\alpha}_{\mathbf{2}} \cap \boldsymbol{\alpha}_{\mathbf{3}}$. Then go to Step 4 below. 

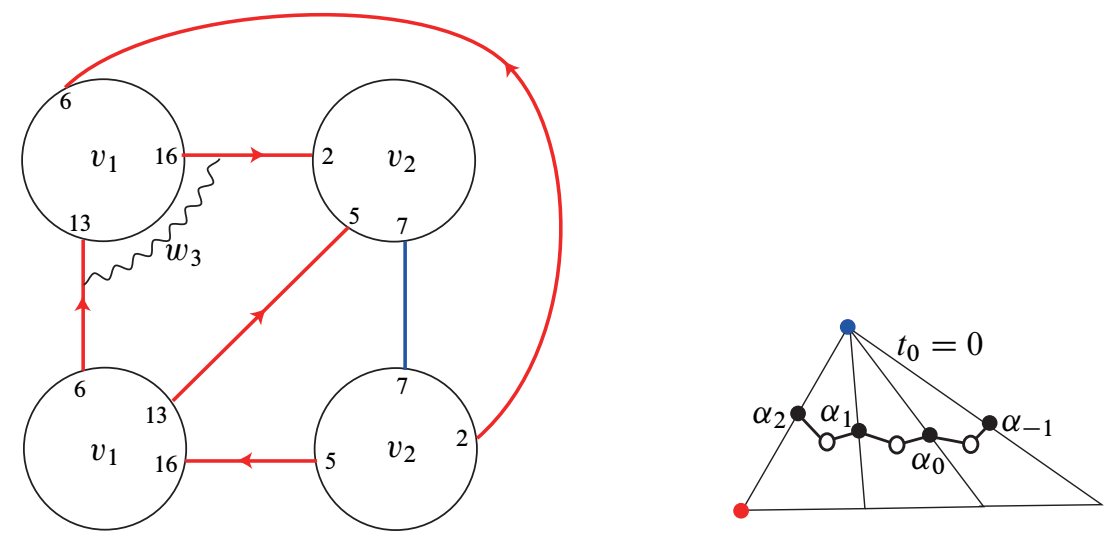

Figure 11: $D_{2}=\left(\partial H ; \boldsymbol{\alpha}_{\mathbf{2}}, \boldsymbol{v}\right)$
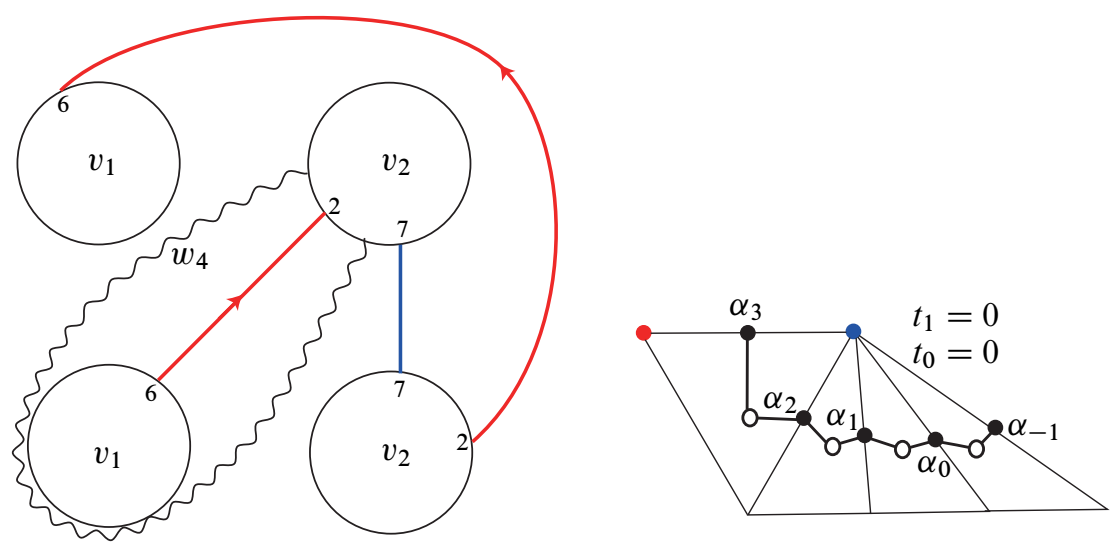

Figure 12: $D_{3}=\left(\partial H ; \boldsymbol{\alpha}_{3}, \boldsymbol{v}\right)$

\section{Step 4}

(4-1) $D_{3}$ is connected, so we go to (4-2).

(4-2) $D_{3}$ has a $\boldsymbol{v}$-wave $w_{4}$ as illustrated in Figure 12. A Heegaard diagram $D_{3}^{\prime}=$ $\left(\partial H ; \boldsymbol{\alpha}_{\mathbf{3}}, \boldsymbol{v}^{\prime}\right)$ obtained by performing the wave transformation is illustrated in Figure 13. We go back to (4-1) after replacing $\boldsymbol{v}$ with a new one.

(4-1) $D_{3}^{\prime}$ is the standard diagram, so we finish the algorithm.

We have found that the binary invariants of the tunnel $\gamma_{1}$ are $\left(s_{2}, s_{3}\right)=\left(t_{1}, t_{0}\right)=$ $(0,0)$. By Proposition $2.10, \operatorname{depth}\left(\gamma_{1}\right)=1$, so the tunnel $\gamma_{1}$ is a $(1,1)$-tunnel from Remark 2.9. 

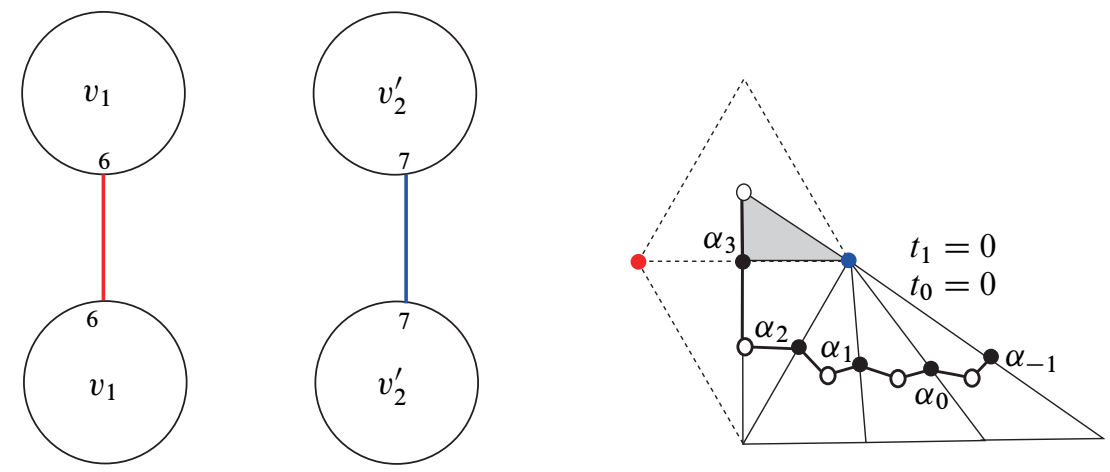

Figure 13: $D_{3}^{\prime}=\left(\partial H ; \boldsymbol{\alpha}_{\mathbf{3}}, \boldsymbol{v}^{\prime}\right)$

\subsection{The tunnel $\gamma_{2}$}

A regular neighborhood $\mathrm{N}\left(K \cup \gamma_{2}\right)$ of $K \cup \gamma_{2}$ is an unknotted genus-2 handlebody, so it can be moved to the standard genus-2 handlebody $H$ in $S^{3}$ by isotopy as illustrated in Figure 14. Let $u_{2}$ be the meridian of $H$ that corresponds to $\gamma_{2}$ and $m_{2}$ a meridian of $H$ that corresponds to a meridian of $K$ as in Figure 14. Cutting $\partial H$ open along meridians $v_{1}$ and $v_{2}$ of $\mathrm{Cl}\left(S^{3}-H\right)$ produces the Heegaard diagram $D_{-1}=\left(\partial H ; \boldsymbol{\alpha}_{-\mathbf{1}}, \boldsymbol{v}\right)$ seen in the left-hand side of Figure 15, where $\boldsymbol{\alpha}_{-\mathbf{1}}=\left\{u_{2}, m_{2}\right\}$ and $\boldsymbol{v}=\left\{v_{1}, v_{2}\right\}$. Now we apply the algorithm to $\gamma_{2}$ by using $D_{-1}$.

\section{Step 0}

(0-1) $D_{-1}$ is connected, so we go to $(0-2)$.

(0-2) $\quad D_{-1}$ has no $\boldsymbol{v}$-wave, so we go to (0-3).

(0-3) $D_{-1}$ has no $m_{2}$-wave, so we go to (0-4).

(0-4) Then $D_{-1}$ has a $u_{2}$-wave $w_{0}$ as illustrated in Figure 15. A Heegaard diagram $D_{0}=\left(\partial H ; \boldsymbol{\alpha}_{\mathbf{0}}, \boldsymbol{v}\right)$ obtained by performing the wave transformation along $w_{0}$ is illustrated in Figure 16, and we go to Step 1 below.

\section{Step 1}

(1-1) $\quad D_{0}$ is connected, so we go to (1-2).

(1-2) $D_{0}$ has no $\boldsymbol{v}$-wave, so we go to (1-3).

(1-3) Then $D_{0}$ has a $\boldsymbol{\alpha}_{0}$-wave $w_{1}$ as illustrated in Figure 16. A Heegaard diagram $D_{1}=\left(\partial H ; \boldsymbol{\alpha}_{1}, \boldsymbol{v}\right)$ obtained by performing the wave transformation along $w_{1}$ is illustrated in Figure 17, and we go to Step 2 below. 

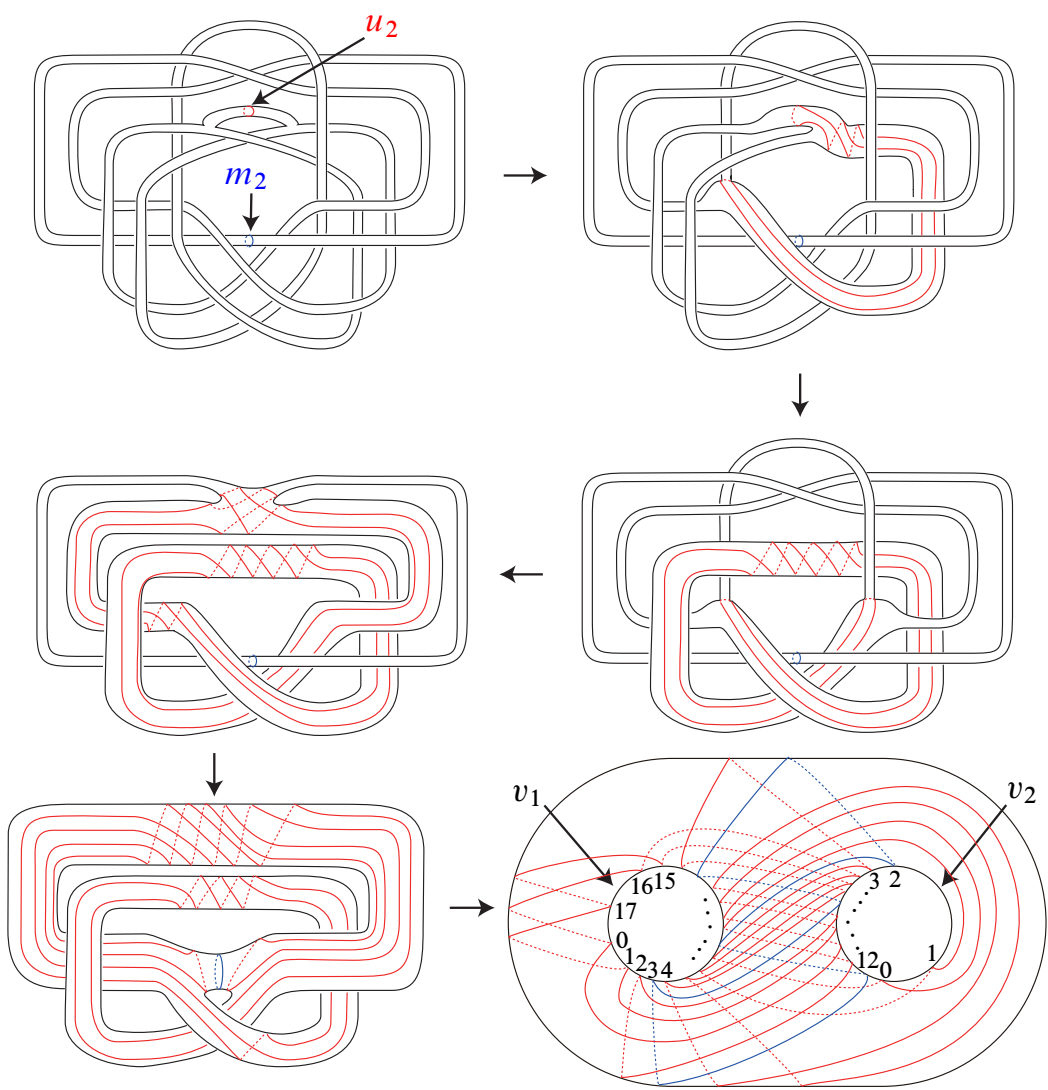

Figure 14: The tunnel $\gamma_{2}$

\section{Step 2}

(2-1) $\quad D_{1}$ is connected, so we go to (2-2).

(2-2) $D_{1}$ has no $\boldsymbol{v}$-wave, so we go to (2-3).

(2-3) Then $D_{1}$ has a $\boldsymbol{\alpha}_{1}$-wave $w_{2}$ as illustrated in Figure 17. A Heegaard diagram $D_{2}=\left(\partial H ; \boldsymbol{\alpha}_{2}, \boldsymbol{v}\right)$ obtained by performing the wave transformation along $w_{2}$ is illustrated in Figure 18, and we go to (2-5).

(2-5) Put $t_{0}=1$ because the unique meridian $\alpha_{0} \cap \alpha_{1}$ does not equal the unique meridian $\boldsymbol{\alpha}_{\mathbf{1}} \cap \boldsymbol{\alpha}_{\mathbf{2}}$. Then go to Step 3 below.

\section{Step 3}

(3-1) $D_{2}$ is the standard diagram and so we finish the algorithm.

We have found that the binary invariant of the tunnel $\gamma_{2}$ is $s_{2}=t_{0}=1$. By Proposition 2.10, depth $\left(\gamma_{2}\right)=2$, so $\gamma_{2}$ is a non- $(1,1)$-tunnel from Remark 2.9. 

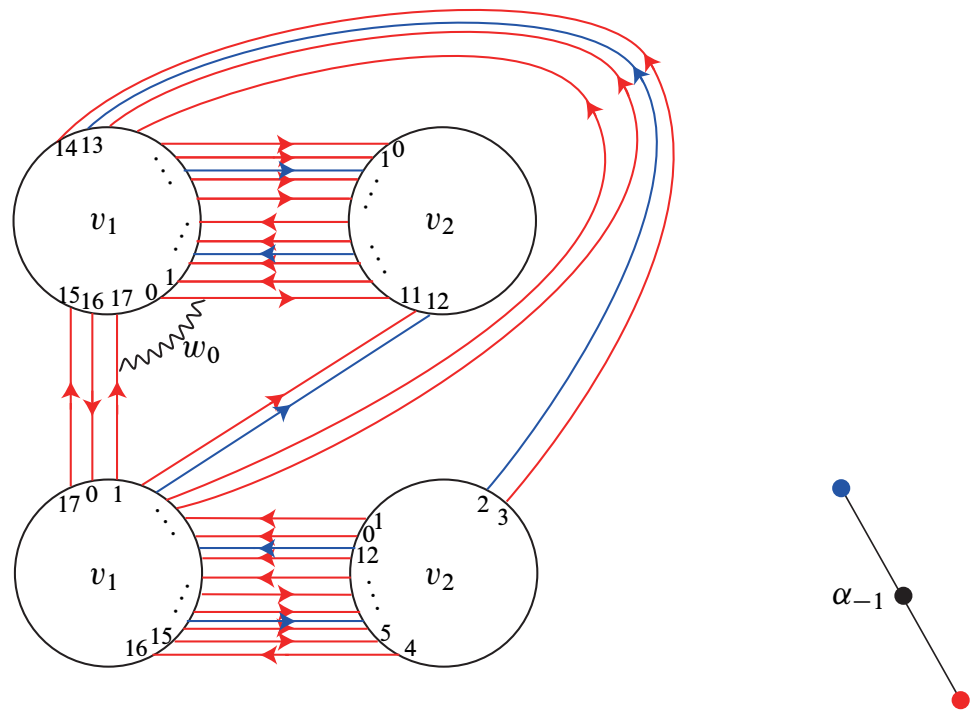

Figure 15: $D_{-1}=\left(\partial H ; \boldsymbol{\alpha}_{-1}, \boldsymbol{v}\right)$
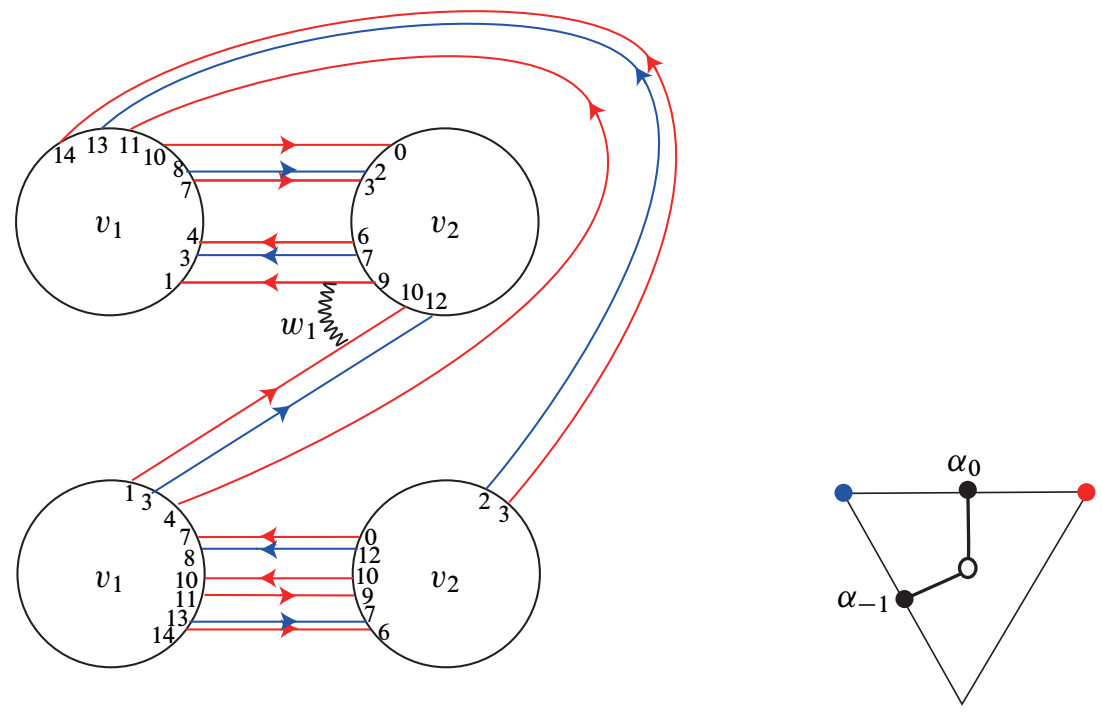

Figure 16: $D_{0}=\left(\partial H ; \boldsymbol{\alpha}_{\mathbf{0}}, \boldsymbol{v}\right)$ 

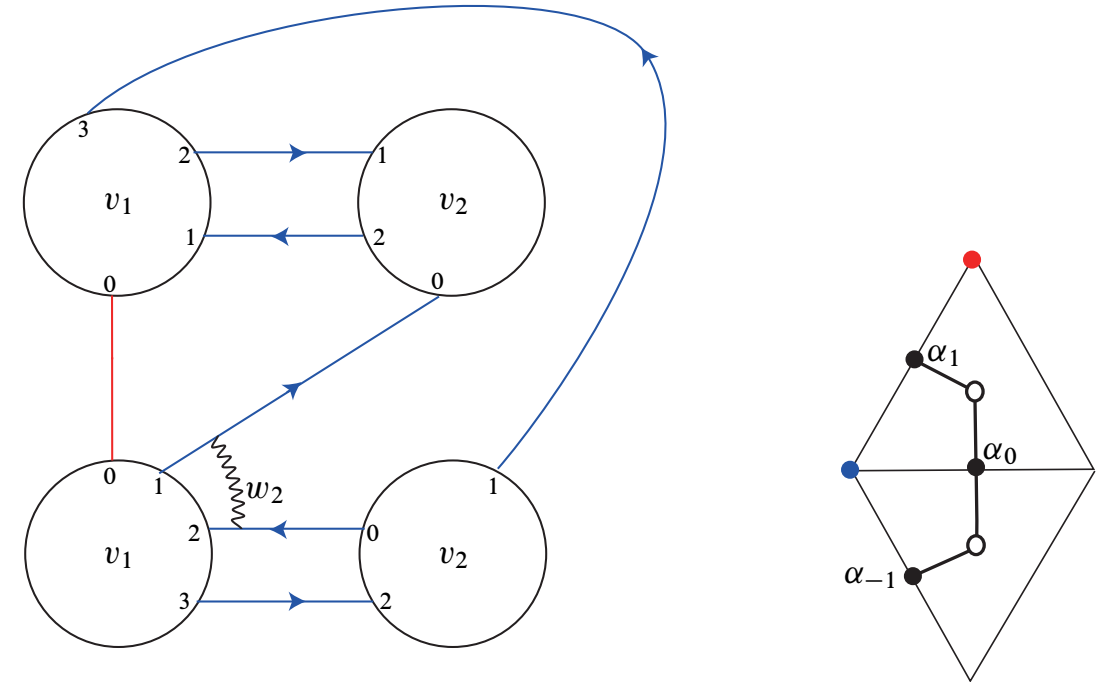

Figure 17: $D_{1}=\left(\partial H ; \boldsymbol{\alpha}_{\mathbf{1}}, \boldsymbol{v}\right)$
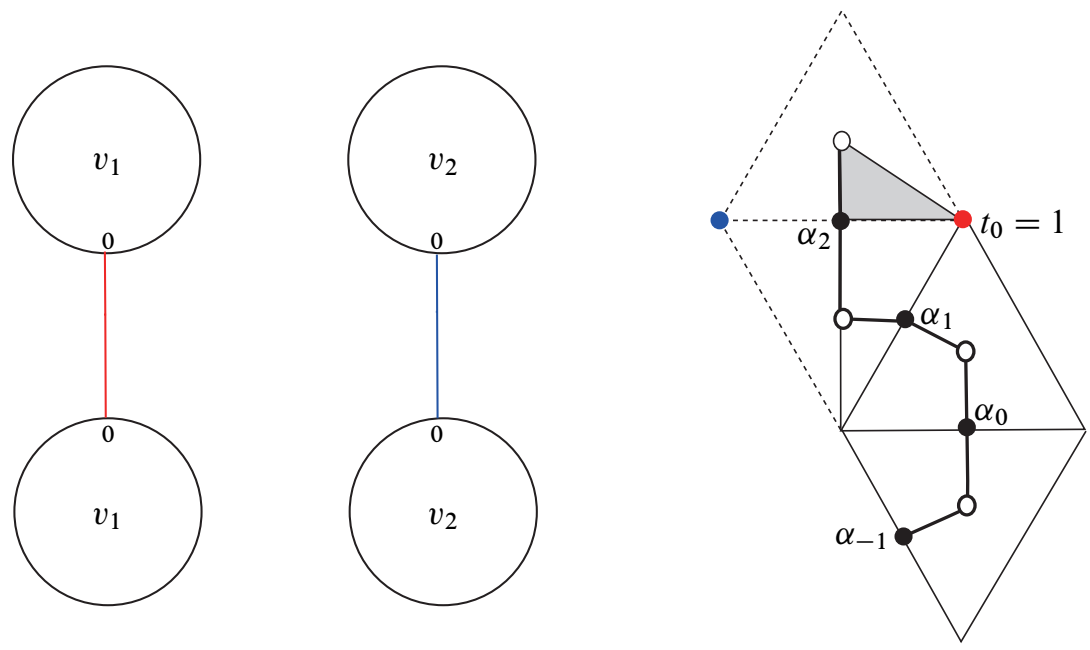

Figure 18: $D_{2}=\left(\partial H ; \boldsymbol{\alpha}_{\mathbf{2}}, \boldsymbol{v}\right)$ 


\section{References}

[1] J Berge, Embedding the exteriors of one-tunnel knots and links in the 3-sphere, unpublished manuscript

[2] S Cho, D McCullough, Cabling sequences of tunnels of torus knots, Algebr. Geom. Topol. 9 (2009) 1-20 MR2471129

[3] S Cho, D McCullough, The tree of knot tunnels, Geom. Topol. 13 (2009) 769-815 MR2469530

[4] S Cho, D McCullough, Tunnel leveling, depth, and bridge numbers, Trans. Amer. Math. Soc. 363 (2011) 259-280 MR2719681

[5] A T Fomenko, S V Matveev, Algorithmic and computer methods for three-manifolds, Math. and its Appl. 425, Kluwer, Dordrecht (1997) MR1486574 Translated from the 1991 Russian original by M Tsaplina and M Hazewinkel and revised by the authors, With a preface by Hazewinkel

[6] H Goda, C Hayashi, Genus two Heegaard splittings of exteriors of 1-genus 1-bridge knots, preliminary version

[7] H Goda, C Hayashi, Genus two Heegaard splittings of exteriors of 1-genus 1-bridge knots arXiv:1009.2134

[8] C M Gordon, J Luecke, Knots are determined by their complements, Bull. Amer. Math. Soc. (N.S.) 20 (1989) 83-87 MR972070

[9] T Homma, M Ochiai, M-o Takahashi, An algorithm for recognizing $S^{3}$ in 3manifolds with Heegaard splittings of genus two, Osaka J. Math. 17 (1980) 625-648 MR591141

[10] K Morimoto, M Sakuma, On unknotting tunnels for knots, Math. Ann. 289 (1991) 143-167 MR1087243

[11] M Ochiai, Heegaard diagrams and Whitehead graphs, Math. Sem. Notes Kobe Univ. 7 (1979) 573-591 MR567245

[12] M Scharlemann, A Thompson, Unknotting tunnels and Seifert surfaces, Proc. London Math. Soc. (3) 87 (2003) 523-544 MR1990938

Department of Mathematics, Imperial College London

London, SW7 2AZ, UK

k.ishihara@imperial.ac.uk

Received: 17 November $2009 \quad$ Revised: 17 May 2011 